EPJ Web of Conferences 66, 01015 (2014)

DOI: $10.1051 /$ epjconf/ 20146601015

(C) Owned by the authors, published by EDP Sciences, 2014

\title{
Towards New Horizons in Ab Initio Nuclear Structure Theory
}

\author{
Robert Roth ${ }^{1, a}$, Angelo Calci ${ }^{1}$, Joachim Langhammer ${ }^{1}$, and Sven Binder ${ }^{1}$ \\ ${ }^{1}$ Institut für Kernphysik, Technische Universität Darmstadt, D-64289 Darmstadt, Germany
}

\begin{abstract}
We present a cross-section of recent advances in ab initio nuclear structure theory, which have changed the horizons of this field. Starting from chiral effective field theory to construct the nuclear Hamiltonian and the similarity renormalization group to further soften it, we address several many-body approaches that have seen major developments over the past few years. We show that the domain of ab initio nuclear structure theory has been pushed well beyond the p-shell and that quantitative QCD-based predictions are becoming possible all the way from the proton to the neutron drip line up into the medium-mass regime.
\end{abstract}

\section{Introduction}

Low-energy nuclear structure and reaction theory underwent a profound change over the past few years. Ab initio nuclear theory, in particular, is swiftly moving towards a systematic theory of nuclear properties for stable and exotic nuclei solidly rooted in QCD. This development is driven by concurring advances in four different areas: (i) the improved quantitative understanding of QCD in the low-energy regime through chiral effective field theory; (ii) a multitude of innovations in many-body theory giving access to new regions of the nuclear chart and new observables; (iii) new algorithms and increased computational resources that help to tackle exceedingly complex problems numerically; (iv) the exciting present and future experimental programs for the study of nuclei far-off stability. Today, the success in ab initio nuclear theory emerges from the interplay of these areas and the transfer of ideas and developments. The collaboration and exchange among these areas is and continues to be vital for the future advancement of low-energy nuclear theory.

In this contribution we give a selective cross-section of developments that are representative for the advances within ab initio nuclear structure theory. We will discuss the status of chiral effective field theory from the practitioners point of view and discuss one of the unitary transformation methods that are used to tame the nuclear Hamiltonian. Then we will address recent achievements in ab initio many-body theory and show selected highlight applications.

\section{Nuclear Hamiltonian}

\subsection{Interactions from Chiral EFT}

Chiral effective field theory (EFT) has become a widely adopted approach to the low-energy dynamics of pions and nucleons capturing the relevant degrees of freedom and symmetries of QCD and thus

\footnotetext{
a e-mail: robert.roth@physik.tu-darmstadt.de
} 
providing a solid link to the underlying theory of the strong interaction $[1,2]$. In the context of the ab initio nuclear structure problem, the nucleon-nucleon (NN) interactions derived from chiral EFT have replaced many of the traditional realistic interactions. There are a number of conceptual and practical reasons for the success of chiral EFT interactions in nuclear structure theory. Among the conceptual reasons are the possibility to derive consistent two-nucleon, three-nucleon $(3 \mathrm{~N})$ and multi-nucleon interactions as well as corresponding electromagnetic and weak current operators and the possibility to systematically improve them by including higher orders of the chiral expansion. Among the practical reasons for the success of chiral EFT Hamiltonians are the high-accuracy of the description of NN phase shifts and light-nuclei properties obtained with chiral NN+3N interactions and the softness of the interactions resulting from the cutoff regularization within the EFT framework.

The chiral EFT Hamiltonian that has been used most widely so far consists of a high-precision $\mathrm{NN}$ interaction at next-to-next-to-next-to-leading order $\left(\mathrm{N}^{3} \mathrm{LO}\right)$ by Entem and Machleidt [3] along with a $3 \mathrm{~N}$ interaction at next-to-next-to-leading order $\left(\mathrm{N}^{2} \mathrm{LO}\right)$ in a local formulation by Navrátil [4] with low-energy constants $c_{D}$ and $c_{E}$ fit to the $A=3$ binding energy and the triton half-life [5]. Obviously, this Hamiltonian does not include $\mathrm{NN}$ and $3 \mathrm{~N}$ interactions at consistent order. The missing $3 \mathrm{~N}$ contributions at $\mathrm{N}^{3} \mathrm{LO}$ have been derived recently $[6,7]$ and a collaborative effort is under way to compute the numerical matrix elements suitable for inclusion in standard many-body approaches (LENPIC collaboration). Apart from the consistent Hamiltonian at $\mathrm{N}^{3} \mathrm{LO}$ several consistent Hamiltonians at $\mathrm{N}^{2} \mathrm{LO}$ are available already, e.g., the Hamiltonians by Epelbaum et al. $[8,9]$ or the recent $\mathrm{N}^{2}$ LO-optimized Hamiltonians [10].

\subsection{Similarity Renormalization Group}

Although the chiral interactions are softer than traditional meson-exchange interactions, their direct inclusion into a configuration-space many-body calculation requires huge and often intractable model spaces in order to account for the correlations induced by the Hamiltonians and to obtain convergence. An efficient tool to tame these correlations and to accelerate the convergence of the subsequent many-body calculation with increasing model-space size are unitary transformations. The so-called similarity renormalization group (SRG) $[11,12]$ has become one of the most popular of these transformations mainly because of its simplicity and flexibility.

The SRG is a continuous unitary transformation formulated in the language of renormalization group flow-equations and governed by a flow parameter $\alpha$. The transformed Hamiltonian $H_{\alpha}$ and any other consistently transformed observable $O_{\alpha}$ is determined by Heisenberg-like first-order differential equations

$$
\frac{\mathrm{d}}{\mathrm{d} \alpha} H_{\alpha}=\left[\eta_{\alpha}, H_{\alpha}\right], \quad \frac{\mathrm{d}}{\mathrm{d} \alpha} O_{\alpha}=\left[\eta_{\alpha}, O_{\alpha}\right], \quad \eta_{\alpha}=(2 \mu)^{2}\left[T_{\mathrm{int}}, H_{\alpha}\right],
$$

with $\eta_{\alpha}$ being the $\alpha$-dependent generator of the transformation. The flexibility of the SRG is rooted in the fact that one is completely free in choosing an appropriate generator $\eta_{\alpha}$ of the transformation as long as it is antihermitian. In the following we restrict ourselves to the definition of the generator as commutator of the intrinsic kinetic energy $T_{\text {int }}$ with the transformed Hamiltonian, which is common in context of nuclear physics (see e.g. [13]). With this choice the Hamiltonian is driven towards banddiagonal form in momentum space. However, other choices of the generator are possible and under investigation.

In order to solve the flow equations numerically, we convert Eq. (1) into matrix-element representation with respect to an $n$-body basis. Technically, this results in a system of coupled ordinary differential equations for the matrix elements of the transformed Hamiltonian $H_{\alpha}$, which can be solved using standard methods. The effect of the SRG transformation on the matrix elements of the NN+3N 
INPC 2013

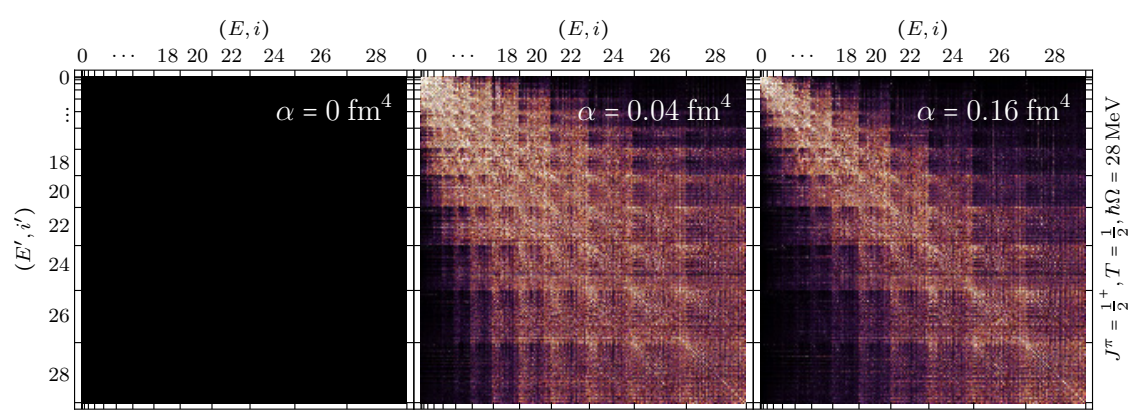

Figure 1. Effect of the SRG evolution on the matrix elements of the NN+3N-full interaction in a three-body harmonic-oscillator Jacobi basis. Plotted are the absolute values of the matrix elements, where light colors represent large values and dark colors represent values near zero.

interaction in the harmonic-oscillator Jacobi basis is illustrated in Fig. 1. The left-hand panel shows the initial interaction, i.e. $\alpha=0 \mathrm{fm}^{4}$, exhibiting large matrix elements even far off the diagonal. The middle and right-hand panels show the SRG-transformed matrix elements for flow parameters $\alpha=0.04 \mathrm{fm}^{4}$ and $0.16 \mathrm{fm}^{4}$, respectively. Evidently, the SRG transformation causes a prediagonalization of the matrix, i.e., a decoupling of the high- and low-energy states and thus improves the convergence of a truncated many-body calculation.

The projection of the operator equations (1) onto an $n$-body Hilbert space automatically discards flow-induced operator contributions beyond the $n$-body level. Thus, when solving the flow equation in, e.g., a three-body matrix representation we will discard possible induced four- and multi-nucleon contributions. The lack of these contributions will destroy the formal unitarity of the SRG transformation and can induce a dependence of the converged energy eigenvalues on the SRG transformation and, more specifically, on the flow parameter $\alpha$. Therefore, the flow parameter provides a valuable tool to quantify the impact of omitted induced many-body contributions and we always assess the dependence of observables on variations of the flow parameter.

Presently, we routinely perform the SRG evolution in the three-body space, i.e., we can include initial and SRG-induced 3N interactions. To reveal the anatomy of the SRG-evolved Hamiltonians, we will use three different truncations: (i) $N N$-only: we start with an initial $\mathrm{NN}$ interaction and keep the two-body terms only; (ii) $N N+3 N$-induced: we start with an initial $\mathrm{NN}$ interaction and keep twoand induced three-body terms; (iii) $N N+3 N$-full: we start with an initial $N N+3 N$ interaction and keep the two- and all three-body terms. First pilot studies of SRG evolutions in four-body space have been successful and will give access to initial and induced $4 \mathrm{~N}$ interactions in the future.

\section{Nuclear Many-Body Problem - Ab Initio}

\section{1 'Light' Nuclei: No-Core Shell Model}

Traditionally, the lower p-shell was the domain of ab initio nuclear structure calculations over the past decade, with the Green's function Monte Carlo (GFMC) approach and the no-core shell model (NCSM) being the most prominent representatives. A series of developments and improvements, both conceptually and computationally, have opened new windows for NCSM-type ab initio calculations extending up into the lower sd-shell.

One of the relevant developments is the importance truncation (IT) of the NCSM model space $[14,15]$. It is based on the fact that many of the NCSM m-scheme states are irrelevant for 


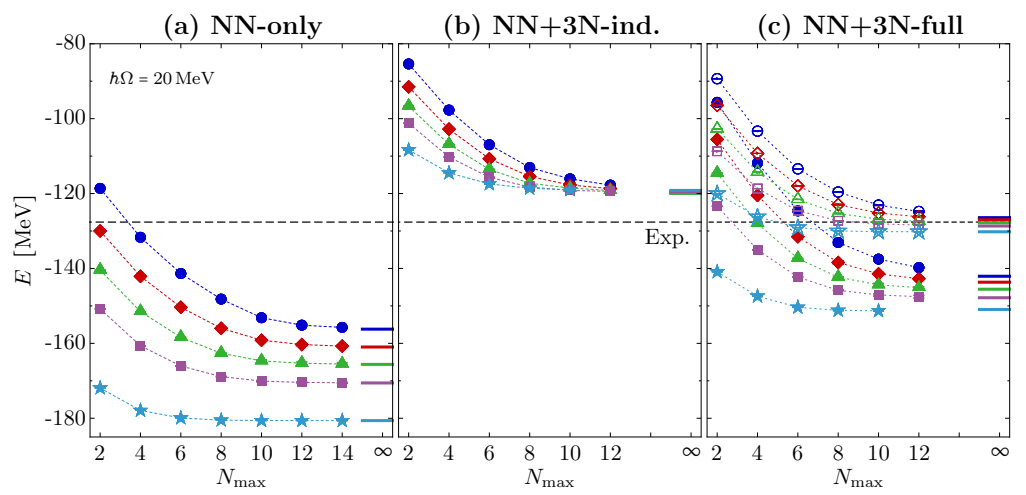

Figure 2. Ground-state energy of ${ }^{16} \mathrm{O}$ obtained in the IT-NCSM as function of model-space truncation $N_{\text {max }}$ for the $\mathrm{NN}$-only (a), the $\mathrm{NN}+3 \mathrm{~N}$-induced (b), and the $\mathrm{NN}+3 \mathrm{~N}$-full Hamiltonian (c). The different symbols correspond to different SRG flow parameters: $\alpha=0.04 \mathrm{fm}^{4}(\bullet), 0.05 \mathrm{fm}^{4}(\bullet), 0.0625 \mathrm{fm}^{4}(\Delta), 0.08 \mathrm{fm}^{4}(\square)$, $0.16 \mathrm{fm}^{4}(\star)$. The filled symbols in panel (c) show results with the chiral $3 \mathrm{~N}$ interaction for $\Lambda_{3 \mathrm{~N}}=500 \mathrm{MeV} / c$, open symbols for $\Lambda_{3 \mathrm{~N}}=400 \mathrm{MeV} / c$.

the description of low-lying eigenstates. Guided by many-body perturbation theory, one can define a measure for the importance of individual basis states and discard states with an importance measure below a threshold value, thus reducing the dimension of the matrix eigenvalue problem. Through a sequence of IT calculations for different thresholds and an a posteriori extrapolation of all observables to vanishing threshold, we can recover the full NCSM results up to extrapolation errors [15]. The ITNCSM together with a novel treatment of the $3 \mathrm{~N}$ matrix elements, based on storing $J T$-coupled matrix elements and their efficient on-the-fly decoupling, enables ab initio calculations including explicit $3 \mathrm{~N}$ interactions in huge model spaces in particular for nuclei in the upper $\mathrm{p}$ - and lower sd-shell [16].

The first ab initio calculations with SRG-evolved chiral $\mathrm{NN}+3 \mathrm{~N}$ interactions in the upper $\mathrm{p}$ shell [17] have demonstrated that sizable SRG-induced 3N contributions emerge from the initial chiral $\mathrm{NN}$ interaction, i.e., unitarily equivalent and flow-parameter independent results can only be obtained at the level of the $\mathrm{NN}+3 \mathrm{~N}$-induced Hamiltonian. This is illustrated in Fig. 2(a) and (b), where the ground state energy of ${ }^{16} \mathrm{O}$ obtained in the IT-NCSM with the NN-only and the NN+3N-induced Hamiltonian is depicted as function of the model-space parameter $N_{\max }$. When including the initial chiral $3 \mathrm{~N}$ interaction, then SRG-induced $4 \mathrm{~N}$ contributions become relevant and are revealed through the flow-parameter dependence of the results with the $\mathrm{NN}+3 \mathrm{~N}$-full Hamiltonian as shown in Fig. 2(c). We emphasize that because of the flow-parameter dependence, the ground-state energies obtained with the SRG-evolved NN+3N-full Hamiltonian do not correspond to the ground-state energies that would be obtained with the initial chiral $N N+3 N$ Hamiltonian - the overbinding visible in Fig. 2(c) could result entirely from the omission of the induced $4 \mathrm{~N}$ interactions, which are known to be repulsive.

One way to reduce the contribution of SRG-induced $4 \mathrm{~N}$ interactions is to reduce the cutoff momentum used in the construction of the initial chiral $3 \mathrm{~N}$ interaction. Already a reduction from the widely-used value of $\Lambda_{3 \mathrm{~N}}=500 \mathrm{MeV} / c$ to $\Lambda_{3 \mathrm{~N}}=400 \mathrm{MeV} / c$ reduces the size of the induced $4 \mathrm{~N}$ contributions significantly. For the latter $3 \mathrm{~N}$ interaction we have readjusted the $c_{E}$ parameter to reproduce the ${ }^{4} \mathrm{He}$ ground-state energy obtained with the bare interaction and kept all other low-energy constants at the values of Ref. [5]. With these parameters the triton binding energy is underestimated by about $150 \mathrm{keV}$ but the half-live will not change. The reduction of the flow-parameter dependence and thus the suppression of the induced $4 \mathrm{~N}$ interactions is clearly visible by comparing the two sets 
INPC 2013
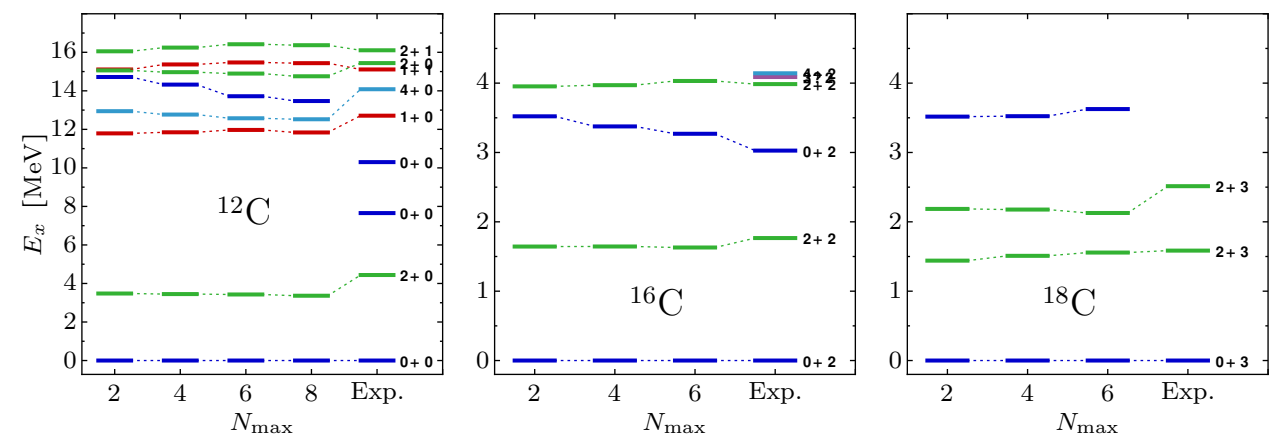

Figure 3. Excitation spectrum of ${ }^{12} \mathrm{C},{ }^{16} \mathrm{C}$, and ${ }^{18} \mathrm{C}$ obtained in the IT-NCSM for $h \Omega=16 \mathrm{MeV}, \alpha=0.08 \mathrm{fm}^{4}$ with the $\mathrm{NN}+3 \mathrm{~N}$-full Hamiltonian for reduced $3 \mathrm{~N}$ cutoff $\Lambda_{3 \mathrm{~N}}=400 \mathrm{MeV} / c$. See Ref. [17] for a related discussion of the ${ }^{12} \mathrm{C}$ spectrum.

of calculations in Fig. 2(c). For $\Lambda_{3 \mathrm{~N}}=400 \mathrm{MeV} / \mathrm{c}$ the SRG-evolved Hamiltonian at the NN+3N-full level is to a good approximation unitarily equivalent to the initial chiral $\mathrm{NN}+3 \mathrm{~N}$ Hamiltonian.

One of the typical applications of the NCSM is the description of spectra and spectroscopy in light nuclei. Within the IT-NCSM we can extend these studies to the upper p- and lower sd-shell with explicit inclusion of chiral $3 \mathrm{~N}$ interactions. As an example for an ongoing research program we present the excitation spectra of selected neutron-rich carbon isotopes in Fig. 3 obtained with the aforementioned chiral $\mathrm{NN}+3 \mathrm{~N}$ Hamiltonian for $\Lambda_{3 \mathrm{~N}}=400 \mathrm{MeV} / c$. We stress that since the Hamiltonian was determined using experimental data up to $A \leq 4$, these calculations in the upper p-shell are completely parameter-free. We find a quite remarkable systematic agreement of the calculated spectra obtained in the IT-NCSM with experimental data, even when approaching the drip line. This is evidence for the predictive power of chiral $\mathrm{NN}+3 \mathrm{~N}$ Hamiltonians beyond the lightest isotopes. These particular calculations are a nice illustration of the interplay and synergy of the four relevant areas mentioned in the introduction: Advances in chiral EFT for NN and $3 \mathrm{~N}$ interactions together with novel innovative methods in many-body theory plus computational resources and algorithmic developments enable ab initio calculations of observables that are just becoming accessible in experiments - like the spectroscopy of ${ }^{16} \mathrm{C}$ and ${ }^{18} \mathrm{C}[18,19]$.

\subsection{Medium-Mass Nuclei: Coupled-Cluster, In-Medium SRG \& Co.}

The regime of medium-mass nuclei is a particularly exciting arena for new developments in manybody theory at the moment. Within a timeframe of a few years several new many-body methods have been introduced into nuclear structure theory that are inspired by approaches in atomic and molecular physics and quantum chemistry. Among those are advanced versions of coupled-cluster theory [20], self-consistent Green's function approaches [21], and the so-called in-medium similarity renormalization group [22]. All of these methods are capable of describing medium-mass and even heavy nuclei with closed sub-shells using $\mathrm{NN}$ interactions. Various extensions to near-closed-shell and to true open-shell nuclei are being explored in all approaches.

For all methods, the inclusion of explicit $3 \mathrm{~N}$ interactions poses significant formal and computational challenges. Recently, we have performed the first large-scale coupled-cluster calculations for ground states of closed-shell nuclei up to ${ }^{56} \mathrm{Ni}$ including explicit $3 \mathrm{~N}$ interaction terms at the singlesand doubles level [23]. These capabilities can be used to benchmark approximate schemes for the inclusion of $3 \mathrm{~N}$ interactions. The most natural and powerful approximation scheme is based on the 


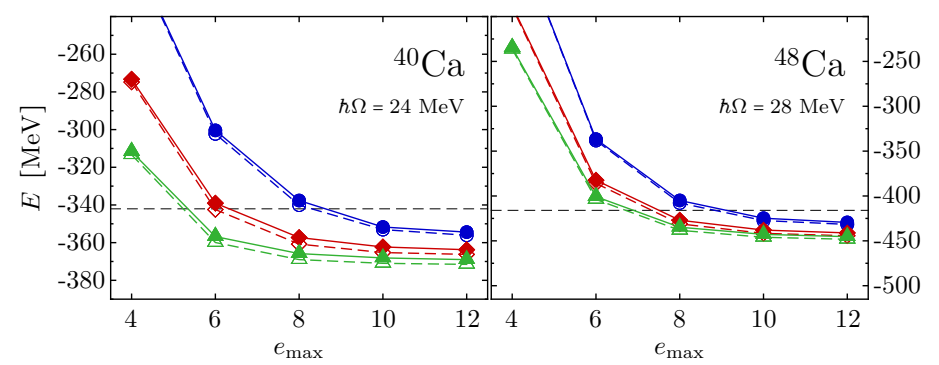

Figure 4. Coupled-cluster singles and doubles calculations for the ground-energies of ${ }^{40} \mathrm{Ca}$ and ${ }^{48} \mathrm{Ca}$ as function of the size of the single-particle basis using the NN+3N-full Hamiltonian $\left(\Lambda_{3 \mathrm{~N}}=400 \mathrm{MeV} / c\right)$ for $\alpha=0.02 \mathrm{fm}^{4}$ $(\bullet), 0.04 \mathrm{fm}^{4}(\bullet)$, and $0.08 \mathrm{fm}^{4}(\bullet)$. Filled symbols correspond to calculations with explicit $3 \mathrm{~N}$ interactions, open symbols to calculations using the normal-ordered two-body approximation.

normal-ordered $3 \mathrm{~N}$ interaction with respect to the coupled-cluster reference state, which is a HartreeFock ground state for the nucleus under consideration. If truncating the normal-ordered $3 \mathrm{~N}$ operator at the two-body level, i.e. in the normal-ordered two-body approximation, parts of the $3 \mathrm{~N}$ interaction are still retained in the zero-, one-, and two-body contributions of the normal-ordered $3 \mathrm{~N}$ operator. Using the normal-ordered two-body approximation results in an enormous simplification of the many-body calculations, since technically only two-body operators need to be handled. Nonetheless, this approximation provides quite accurate results. From the direct comparison of the $\mathrm{CC}$ results with explicit $3 \mathrm{~N}$ interactions to the ones using the normal-ordered two-body approximation shown in Fig. 4 we can conclude that both calculations agree to better than $1 \%$ while the computational cost is reduced by two orders of magnitude in the latter approach [23, 24].

Normal-ordering in the single-reference or more advanced multi-reference formulations is also at the heart of the in-medium SRG. It combines an SRG evolution in many-body space tailored to decouple particle-hole excitations from the reference state with the normal-ordering approximation to absorb induced many-body terms into lower-rank interactions. In contrast to the free-space SRG, discussed in Sec. 2.2, which is used to pre-condition the Hamiltonians for a subsequent many-body calculation, the in-medium SRG directly provides the ground-state energy if the many-body evolution is driven until the reference state is completely decoupled from particle-hole excited many-body states [26]. The expectation value of the evolved Hamiltonian with the reference state, which is just the normal-ordered zero-body contribution of the reference state, yields the ground-state energy of the system.

We demonstrated the power and accuracy of the in-medium SRG for open and closed-shell oxygen isotopes very recently [25]. Using the Hamiltonians discussed above, we computed the ground-state energy of the even oxygen isotopes from proton to neutron drip line in the IT-NCSM, the in-medium SRG, and for the closed sub-shells in coupled-cluster theory up to the doubles level and including triples effects. The results are summarized in Fig. 5. The ab initio IT-NCSM calculations, which do not retain any explicit truncation as the importance truncation is removed though an a posteriori extrapolation, are computationally very demanding. Nevertheless, we were able to perform calculation up to $N_{\max }=12$ even for ${ }^{26} \mathrm{O}$, which is clearly beyond the range of the conventional NCSM. The IT-NCSM results serve as a reference point for the in-medium SRG and coupled-cluster calculations, which both use the normal-ordered two-body approximation to include the $\mathrm{NN}+3 \mathrm{~N}$ Hamiltonians and different truncations in the many-body expansion. 
(a) $\mathrm{NN}+3 \mathrm{~N}$-ind.

(b) $\mathrm{NN}+3 \mathrm{~N}-$ full

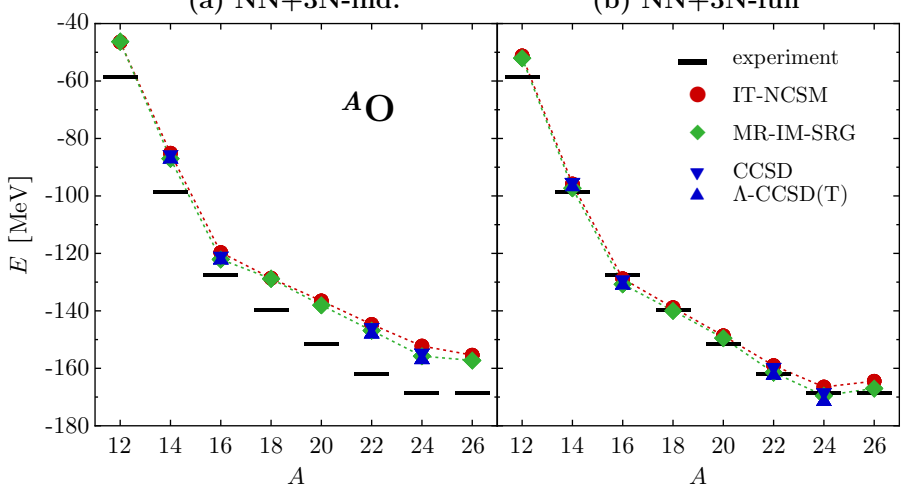

Figure 5. Ground-state energies for the even oxygen isotopes obtained with the IT-NCSM $(\bullet)$, the multireference in-medium SRG $(\diamond)$, and the coupled-cluster method with singles and doubles $(\boldsymbol{\nabla})$ and with the $\Lambda$ $\operatorname{CCSD}(\mathrm{T})$ triples corrections $(\boldsymbol{\Delta})$. Panel (a) shows results for the $\mathrm{NN}+3 \mathrm{~N}$-induced Hamiltonian at $\alpha=0.08 \mathrm{fm}^{4}$, panel (b) with the $\mathrm{NN}+3 \mathrm{~N}$-full Hamiltonian with $\Lambda_{3 \mathrm{~N}}=400 \mathrm{MeV} / c$ and $\alpha=0.08 \mathrm{fm}^{4}$. Adapted from [25].

The agreement among the different methods shown in Fig. 5 is remarkable - within the expected uncertainties, the methods agree with each other. This proves that the many-body problem is well under control and that calculations with quantified uncertainties are possible now, even in this mass region. A second remarkable point is the agreement of the results for the $\mathrm{NN}+3 \mathrm{~N}$-full Hamiltonian with the experimental ground-state energies. This proves that the chiral Hamiltonians contain the physics relevant for a quantitative ab initio description of nuclei at extreme isospins. As has been pointed out in valence-space shell model calculations [27] and coupled-cluster calculation with phenomenological $3 \mathrm{~N}$ effects [28], the $3 \mathrm{~N}$ interaction is necessary to reproduce the drip line at ${ }^{24} \mathrm{O}$. Again we stress that these are parameter-free calculations using a Hamiltonian that was completely determined in $A \leq 4$ systems.

The corresponding calculations with in-medium SRG and coupled-cluster theory for isotopic chains of heavier elements, in particular the calcium and nickel isotopic chains, will be available soon. Work towards the description of excited states and spectroscopic observables is under way and promises to provide a complete ab initio picture of nuclear structure also in the medium-mass regime.

\section{Conclusion \& Outlook}

$\mathrm{Ab}$ initio nuclear structure theory has expanded its horizons drastically over the past years. It has ventured into hitherto inaccessible regions of the nuclear chart and strengthened its foundations in low-energy QCD. There is an unprecedented number and diversity of innovations aiming to further nurture ab initio theory and to connect it to the exciting probes into nuclear structure and reaction phenomena that present and future experiments offer.

The coming years will see further important advances. Keywords that characterize some of our future activities are: consistency, error quantification, continuum and reactions, and hypernuclear structure. We will work towards the consistent inclusion of the inputs from chiral EFT into nuclear structure physics, this includes consistent chiral Hamiltonians at $\mathrm{N}^{3} \mathrm{LO}$ and the corresponding electroweak current operators at the two-body level with consistent SRG transformation and cutoffs. We have started already to rigorously quantify the uncertainties of the various many-body frameworks, a next step will be the systematic propagation of the errors from the chiral EFT inputs (due to truncations 
and uncertainties in the low-energy constants) to nuclear structure observables. The ab initio description of continuum effects and reaction processes is advancing very quickly. Recently, we presented first scattering calculations in the resonating-group method combined to the NCSM with NN+3N interactions at the same level of sophistication as in the structure calculation [29]. Finally, the tools developed in ab initio nuclear structure can be generalized to include additional constituents. In particular the inclusion of hyperons, leading to ab initio hypernuclear structure theory, opens new fields of application. The future of ab initio nuclear theory is bright and exciting!

Acknowledgements: Numerical calculations were performed at the Jülich Supercomputing Centre, the LOEWE-CSC, and the National Energy Research Scientific Computing Center (NERSC), which is supported by the U.S. DOE under Contract No. DE-AC02-05CH11231. Supported by the Deutsche Forschungsgemeinschaft through SFB 634, the Helmholtz International Centre for FAIR (HIC for FAIR), and by the BMBF-FSP 302 'NUSTAR.de' (06DA7074I).

\section{References}

[1] R. Machleidt, D.R. Entem, Phys. Rep. 503, 1 (2011)

[2] E. Epelbaum, H.W. Hammer, U.G. Meißner, Rev. Mod. Phys. 81, 1773 (2009)

[3] D.R. Entem, R. Machleidt, Phys. Rev. C 68, 041001(R) (2003)

[4] P. Navrátil, Few Body Syst. 41, 117 (2007)

[5] D. Gazit, S. Quaglioni, P. Navratil, Phys. Rev. Lett. 103, 102502 (2009)

[6] V. Bernard, E. Epelbaum, H. Krebs, U.G. Meißner, Phys. Rev. C 77, 064004 (2008)

[7] V. Bernard, E. Epelbaum, H. Krebs, U.G. Meissner, Phys.Rev. C84, 054001 (2011)

[8] E. Epelbaum, W. Glöckle, U.G. Meißner, Nucl. Phys. A 747, 362 (2005)

[9] E. Epelbaum, A. Nogga, W. Glöckle, H. Kamada et al., Phys. Rev. C 66, 064001 (2002)

[10] A. Ekström, G. Baardsen, C. Forssén, G. Hagen, et al., Phys. Rev. Lett. 110, 192502 (2013)

[11] R. Roth, T. Neff, H. Feldmeier, Prog. Part. Nucl. Phys. 65, 50 (2010)

[12] S.K. Bogner, R.J. Furnstahl, A. Schwenk, Prog. Part. Nucl. Phys. 65, 94 (2010)

[13] S.K. Bogner, R.J. Furnstahl, R.J. Perry, Phys. Rev. C 75, 061001(R) (2007)

[14] R. Roth, P. Navrátil, Phys. Rev. Lett. 99, 092501 (2007)

[15] R. Roth, Phys. Rev. C 79, 064324 (2009)

[16] R. Roth, A. Calci, J. Langhammer, S. Binder, arXiv:1311.3563 (2013)

[17] R. Roth, J. Langhammer, A. Calci, S. Binder, P. Navrátil, Phys. Rev. Lett. 107, 072501 (2011)

[18] M. Petri, S. Paschalis, R.M. Clark, P. Fallon et al., Phys. Rev. C 86, 044329 (2012)

[19] P. Voss, T. Baugher, D. Bazin, R.M. Clark et al., Phys. Rev. C 86, 011303(R) (2012)

[20] G. Hagen, T. Papenbrock, D.J. Dean, M. Hjorth-Jensen, Phys. Rev. C82, 034330 (2010)

[21] V. Somà, T. Duguet, C. Barbieri, Phys. Rev. C 84, 064317 (2011)

[22] K. Tsukiyama, S.K. Bogner, A. Schwenk, Phys. Rev. Lett. 106, 222502 (2011)

[23] S. Binder, J. Langhammer, A. Calci, P. Navrátil, R. Roth, Phys. Rev. C 87, 021303 (2013)

[24] R. Roth, S. Binder, K. Vobig, A. Calci et al., Phys. Rev. Lett. 109, 052501 (2012)

[25] H. Hergert, S. Binder, A. Calci, J. Langhammer, R. Roth, Phys. Rev. Lett. 110, 242501 (2013)

[26] H. Hergert, S.K. Bogner, S. Binder, A. Calci et al., Phys. Rev. C 87, 034307 (2013)

[27] T. Otsuka, T. Suzuki, J.D. Holt, A. Schwenk, Y. Akaishi, Phys. Rev. Lett. 105, 032501 (2010)

[28] G. Hagen, M. Hjorth-Jensen, G.R. Jansen, et al., Phys. Rev. Lett. 108, 242501 (2012)

[29] G. Hupin, J. Langhammer, P. Navrátil, S. Quaglioni et al., Phys. Rev. C 88, 054622 (2013) 